\title{
The Research and Implementation of Inserting Multi-row Records Based on Relational Database
}

\section{Luo Hongmei}

Jiangxi vocation college of Ahead Software, Nanchang, 330041, China

Keywords: Relational Database, SQL Statement, INSERT, SELECT, UNION ALL

\begin{abstract}
In the process of program development, it is often necessary to insert multi rows of explicitly specified values into one table of relational database at a time, and we hope to achieve this insertion by only one statement. But in the standard SQL statement, there is no such a SQL syntax. In this paper, through the study of 'INSERT ', 'SELECT' and 'UNION ALL' statements, a simple and effective implementation method is given.
\end{abstract}

\section{Introduction}

For software developers, the T-SQL language should be familiar with all three types of statements, that is, DDL (data definition language), DML (data manipulation language), DCL (Data Control Language). The INSERT statement is a statement that belongs to DML to insert data.

The software developers can not avoid inserting multi-row records into a table in a database,then many people think directly of writing out multiple statements of 'INSERT VALUES', separated by semicolons. But in executing these statements, if one of the statements raises an error, we need to find out which one went wrong from multiple statements. After correcting the error, we need to execute the unexecuted statement again. This operation is very low efficiency. There is also a syntax of inserting multi rows in a standard SQL statement by the statement of "INSERT ... SELECT", the premise is that the multi-row datas must be stored in the existing data source. Some of relational databases can also insert multi-row datas directly by the statement of "INSERT ... VALUES", as long as multi-row datas are separated by comma, but this syntax is not supported by SQL SERVER database low than version 2005 and the Oracle database, it is not universal.

In view of the limitations of these INSERT statements, a solution of how to insert multi rows of explicitly specified values into a table by only one statement is proposed,and this solution is universal in most of the relational databases.

\section{The Implementation of Inserting Multi-row records}

\subsection{The basic syntax of the INSERT statement}

INSERT inserts new row record into an existing table. The INSERT ... VALUES forms of the statement insert rows based on explicitly specified values. The INSERT ... SELECT form inserts rows selected from another table or tables.

\subsubsection{INSERT ... VALUES}

In SQL statement, the common using of fill datas into a table is to use the INSERT VALUES statement.The basic syntax is as below:

INSERT INTO < table_name>

[(field1, field 2,..., field n)]

VALUES (value1, value $2, \ldots$, value $n$ )

In this statement, the VALUES clause is used to provide the explicitly specified values that are inserted into the table, and the order of the values must be as same as the sequence of the columns. The main feature is that "only one row record can be inserted at one time". 


\subsubsection{INSERT ... SELECT}

The above described about INSERT statement can only insert one row of record into a table at one time. In practical applications, we often need to insert multi rows at one time. With INSERT ... SELECT, you can quickly insert many rows into a table from the result of a SELECT statement, which can select from one or many tables. The basic syntax is as below:

INSERT INTO < table_name $>$

[(field1, field 2,..., fieldn)]

SELECT column list

FROM table_source

WHERE search conditions

The values of the INSERT statement is the resultset generated by the SELECT statement, but it is limited that the inserted data must be obtained from other data sources, it can't insert multi rows of explicitly specified values in one time.

\subsection{UNION ALL}

Multiple different queries will generate multiple different resultset, but in practical applications, we want to join the multiple different resultset. Then we need to use the statement of UNION ALL, It can join multiple resultset into one resultset.

UNION ALL is used to combine the resultset from multiple SELECT statements into a single resultset. Selected columns listed in corresponding positions of each SELECT statement should have the same datatype.

The basic syntax of UNION ALL is as below:

SELECT select_list From table_source [WHERE search conditions]

$\{\mathrm{UNION}[\mathrm{ALL}]\}$

SELECT select_list From table_source [WHERE search conditions]

[ $\{$ UNION $[\mathrm{ALL}]\}$

SELECT select_list From table_source [WHERE search conditions]]

\subsection{Build explicitly specified values into multi rows by SELECT}

Generally, we are familiar with SELECT statements that can be used to query data from tables and generate resultset, but how to generate resultset by the explicitly specified values that doesn't store in tables? Take the MySQL database as an example, the reference syntax is as below:

SELECT constant_list

UNION ALL SELECT constant_list

[UNION ALL SELECT constant_list]

For example, write down a SQL statement in the MySQL query window:

SELECT 1, 'Jiangxi' UNION ALL

SELECT 2, 'Ahead' UNION ALL

SELECT 3,'Luo' UNION ALL

SELECT 4,'Hongmei'

Generate query results as Figure 1: 


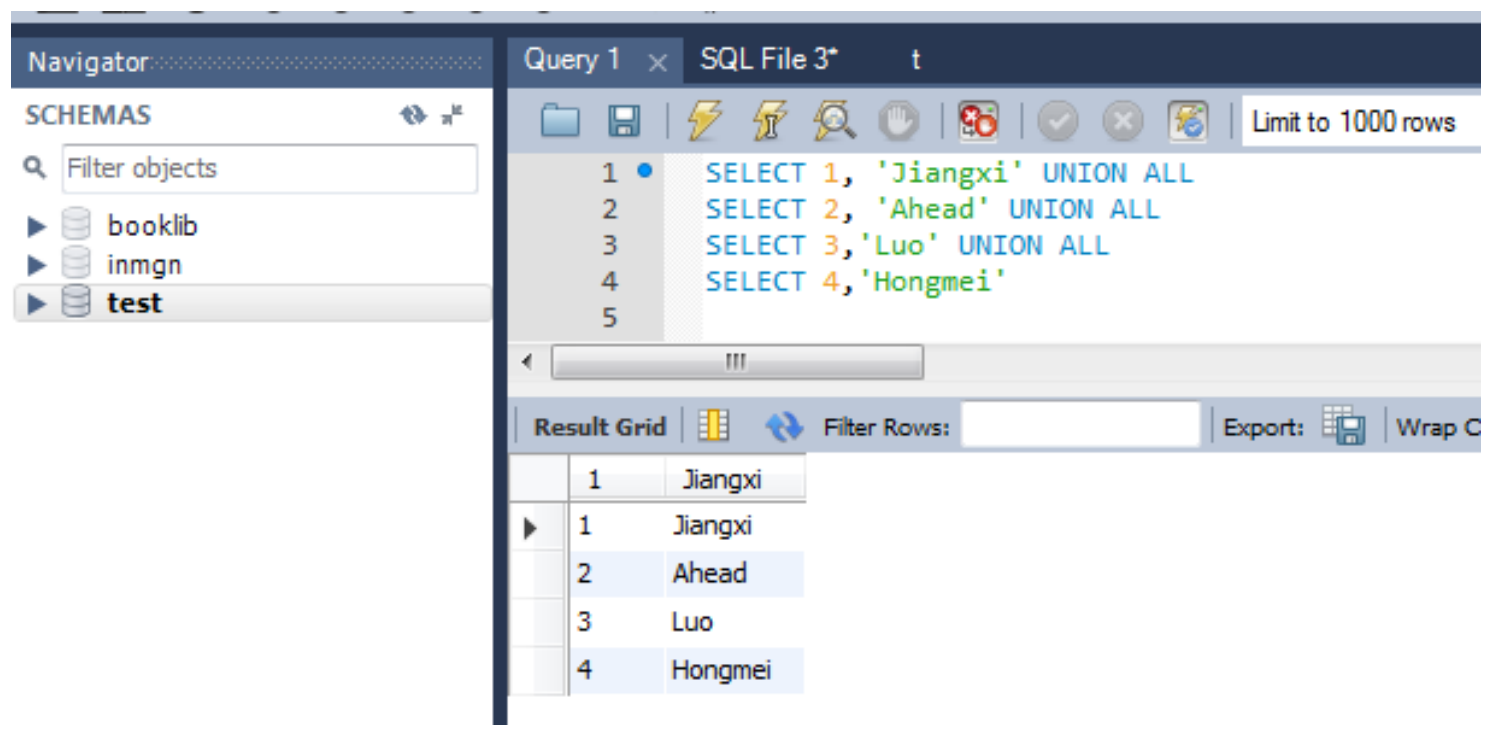

Figure 1 The resultset of query

\subsection{Combine with INSERT, SELECT and UNION ALL to insert multi rows of explicitly specified values}

In combination with 'INSERT', 'UNION ALL' and 'SELECT' statement, this paper give a solution that how to insert multi rows of explicitly specified values at one time.Take the MySQL database as an example, the solution is as follows.

First, we create a table in MySQL database named "T". such as table 1:

Table 1 The structure of table $\mathrm{T}$

\begin{tabular}{|c|c|c|c|}
\hline Field Name & Data Type & length & describe \\
\hline ID & int & & Primary key \\
\hline NAME & VARCHAR & 50 & \\
\hline
\end{tabular}

Second, using the knowledge points described above: 'SELECT' statement make up one row of explicitly specified values, 'UNION ALL' statement join the multiple resultset into one resultset, then insert the combined resultset into table by 'INSERT SELECT' statement. Now the purpose of this article studying is reached.

The reference statement is as below:

\section{INSERT INTO T}

SELECT 1, 'Jiangxi' UNION ALL

SELECT 2, 'Ahead' UNION ALL

SELECT 3, 'Luo' UNION ALL

SELECT 4, 'Hongmei'

The above SQL statement is one INSERT statement, when we execute this statement in the SQL query window,it will display the result as Figure 2: 


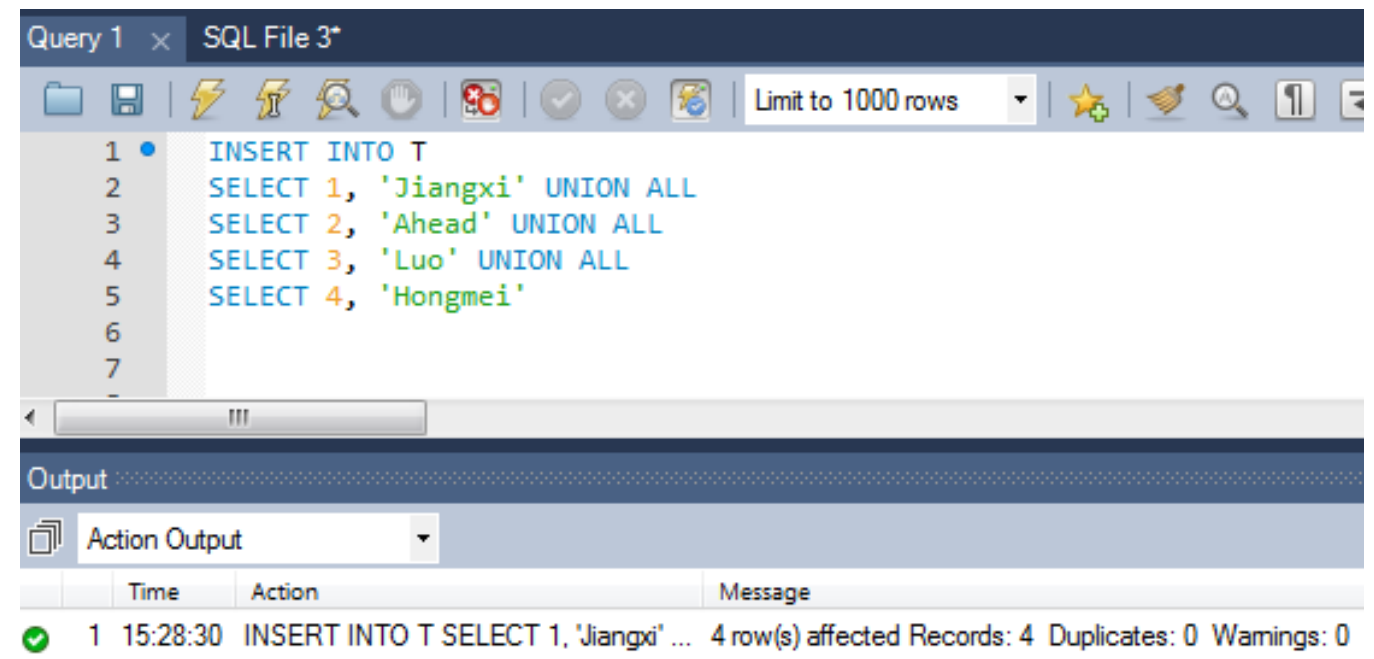

Figure 2. The execution result of INSERT

Now, open the table named $\mathrm{T}$ can see that the 4 rows of records has been inserted in one time. Such as Figure 3:

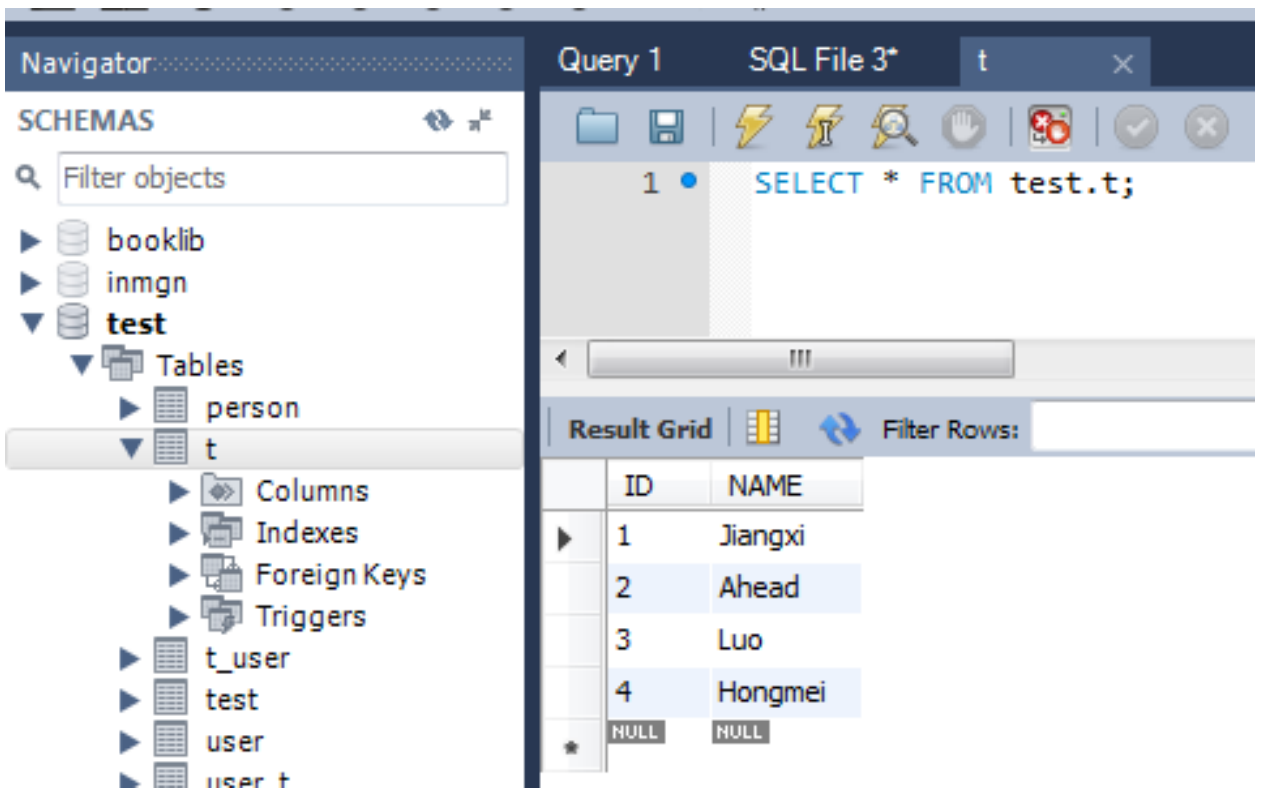

Figure 3. Display the data of Table T

\section{Conclusion}

In this paper,INSERT and SELECT and UNION ALL are combined to give a method of inserting multiple data in one INSERT statement at one time, and this method is universal in the current mainstream relational database.The above reference statement, are also applicable in the SQL Server database.

Similarly,if we need to implement this effect in the ORACLE database, the reference statement is as below:

\section{INSERT INTO T}

SELECT 1, 'Jiangxi' FROM dual UNION ALL

SELECT 2, 'Ahead' FROM dual UNION ALL

SELECT 3, 'Luo' FROM dual UNION ALL

SELECT 4, 'Hongmei' FROM dual 
In this statement,Dual is a virtual table,used to complete sql statement,we can select anything from dual(dummy). The statement of SELECT making up one row of explicitly specified values need to be with the help of the table named dual.

\section{References}

[1] ISO22901: Road Vehicles - Open diagnostic data exchange, 2007.

[2] Beginning SQL Server 2005 for Developers Robin Dewson 2006- Springer 10.1007 /978- 14302-0124-3_5

[3] Pro MySQL Michael Kruckenberg 2005-Springer 10.1007/978-1-4302-0048-2_11

[4] Oracle ${ }^{\circledR}$ Database 2 Day DBA 11g Release 2(11.2) E10897-09, February 2012

[5] Oracle ${ }^{\circledR}$ Database Concepts 11g Release 2(11.2) E25798-01, September 2011 